\title{
Global Perspectives: assessing the impact of new technology
}

The recent Adelaide ASOHNS meeting was entitled "Global Perspectives" for an important reason - as a geographically remote island (albeit a big one!), we often lose track of what is happening elsewhere in the world, or make inaccurate conclusions from the information we do gain from overseas. It was our intention to make this year's Annual Scientific Meeting a forum where we could hear what was happening elsewhere, so we could work out where Australian practice sits in the huge spectrum of the way our specialty is practised around the globe. In the USA, expensive new technology such as trans-oral robotic surgery and fibre-optic laser delivery systems are being funded and integrated into mainstream practice with little or no real evidence of benefit, whereas in the UK, purchasers are declining to fund operations such as adenotonsillectomy on the basis that these procedures deliver little long-term benefit to the community. Managed health care in the USA is now the norm and surgeons have to obey the protocols and guidelines set down by the insurers in order to get paid for the procedures they perform. In Australia, we have so far been spared the ravages of such an aggressive approach to health care management, but most of us are in little doubt that it is only a matter of time before the government and the private insurance sector try to introduce such measures on our home soil.

In Australia, we are actually in a unique position; we have a health care system that is relatively efficient, yet is not so starved of funds that we cannot implement effective new technology. We have a mixture of public and private practice that provides an ideal opportunity for new technology to be assessed in a very rigorous and effective way, something that is not always possible in cash restricted systems, such as the UK, nor in North America where new technology is king and surgical trials become almost impossible to perform.

There are many surgeons in Australia who believe that it is unethical to randomise patients to two different types of surgery or to surgery versus conservative management. It is not unethical to do so however, when a new procedure or technology has not yet established itself into widespread clinical practice. Robotic surgery, balloon sinus dilatation and implantable hearing aids are three such examples that spring to mind. It would be perfectly possible for Australia to perform trials on these three new technologies, which would be almost impossible to perform in many other countries of the world. If we are able to demonstrate cost effectiveness and improved patient outcomes, then we will be in a much better position to be able to fight back when either the public or private treasurers attempt to restrict the introduction of such technology as a cash saving measure.

"Knowledge is Power" is an old saying but it is certainly true when it comes to defending surgical practice. Whilst I am certainly not a proponent of randomised controlled trials in every aspect of what we do, I do feel that prospective evaluation, in combination with RCTs where appropriate, provides much higher levels of evidence than is currently available for many new technological interventions.

Reflecting on the talks at this year's Annual Scientific Meeting, I believe that Australia can take the lead on the world OHNS stage by making sure we do not simply jump on the band wagon with new technology, but by being the country that either provides the evidence that it is effective and therefore encourages introduction, or establishes that a new technology is not cost effective and takes the lead in abandoning its use.

Whilst many individual research units have been extremely successful over the last decade, perhaps now is the time to set up more collaborative projects between departments, cities and states. The funding of such groups and projects is always a concern but national research trial groups have been established in other specialties, obtaining money from a variety of funding sources including Government. In my personal opinion, the time is right for our community to be proactive in pushing for a similar collaborative research body for Australian ENT trials.

A SIMON CARNEY ASOHNS Supplements Editor scarney@ent-surgery.com Adelaide May 2012 